\title{
A mixed finite element for interlaminar stress computation
}

\author{
Yi-Bing Shi \\ Department of Materials Science and Engineering, University of Michigan, Ann Arbor, Michigan 48109, USA \\ $\boldsymbol{\&}$ \\ Hao-Ran Chen \\ Research Institute of Engineering Mechanics, Dalian University of Technology, People's Republic of China
}

\begin{abstract}
A mixed finite element method (MFEM) aiming at solving the problem of three-dimensional stress analysis of multi-layer composite laminates with a high accuracy is presented. The approach, which is based on the global-local laminate variational model, proposes a mixed use of a hybrid stress element within a high precision stress solution region in the thickness direction of the laminate and a conventional displacement finite element in the remaining. This results in a reduction of the overall computation time while maintaining the solution precision in the area(s) of interest, normally being certain interface(s) within a laminate. A formulation of a 49 stress parameter hybrid stress element in conjunction with a 42 degree of freedom iso-parametric displacement element is given. The quality of the hybrid stress element is assured by a stiffness matrix eigenvalue analysis. Example computations of laminate cylindrical bending and the classical free-edge problem have shown the feasibility of the MFEM approach and the convergence of the specific $49 \beta-42 q$ formulation.
\end{abstract}

\section{INTRODUCTION}

Delamination of composite laminates has been identified as a major life-limiting mode of failure in fibre composite applications and has become an important factor to be considered in designing laminate structures. Transverse loading or geometric discontinuity induced interlaminar stresses are usually responsible for delaminations, and analysis of such stresses is required to understand the failure of laminates and provide support to experimental failure mechanism characterizations.

The pioneer attempt to solve the laminate interlaminar stress problem was the study of the free-edge model ${ }^{1}$ by Pipes and Pagano which revealed the three-dimensional nature of the stress state near free edges, and the lamination of the two-dimensional classical laminate theory (CLT). The investigation of interlaminar stress has since extended to the evaluation of fracture mechanics parameters ${ }^{2}$ and laminates with more general geometries. $^{3}$

Because of the complexity of interlaminar stress analyses, the finite element method as a flexible stress analysis tool has been widely used. Normally, at least one layer of elements is required per lamina to model the ply to ply change of elastic properties. Experience has shown that the large amount of computational work by such an approach denies its applications to the analysis of laminates used in practical engineering structures, normally having multiple layers. To meet this special challenge of multilayer interlaminar stress analysis, efforts have been made to construct high order hybrid stress elements $^{4.5}$ and implement computational time reduction schemes. ${ }^{6.7}$ Since solution precision and computational time compete with each other, compromises have to be made to achieve effectively a reliable and efficient stress analysis.

Two associated laminate variational models ${ }^{8.9}$ have been proposed as a general theoretical basis for the class of interlaminar stress analysis problems, and successful applications to the edge delamination problem through a semi-analytical approach have been reported. ${ }^{8-10}$

The objective of the present research is to establish a numerical approach that takes into consideration both the efficiency and solution 
precision of the analysis. A mixed finite element (MFEM) approach is proposed as a numerical application of the global-local variational model ${ }^{9}$ by Pagano and Soni for multi-layer laminate analysis, and the feasibility of the MFEM concept is demonstrated by a specific formulation and numerical examples.

\section{THEORY AND GENERAL FORMULATION OF THE MFEM}

\subsection{Global-local variational model and mixed finite element concept}

The global-local variational model $^{9}$ materializes its ability to tackle the multi-layer laminate analysis problem, which normally involves an intractable amount of work, by dividing the laminate into two distinctive types regions throughout the thickness. Complementary and potential energy principles are employed in the two kinds of regions, named the local and global domains, respectively. The unique feature of this approach is that it enables independent stipulations of a high order stress field in the local region and a plate type displacement distribution in the global region. The objective is achieved to minimize the work in obtaining precise stress solution at the particular laminate interface(s) of concern in the local region.

A mixed finite element concept is proposed in this study to translate this merit of the global-local variational model into the finite element method, and a formulation is presented to illustrate its feasibility. The term 'mixed' is adopted here to refer to a combined use of a hybrid stress element in the local domain (region of high stress solution precision) and a multi-layer iso-parametric displacement element in the global domain.

The functional of the global-local model is: ${ }^{9}$

$$
\begin{aligned}
F(u, \sigma)= & \int_{V_{\mathrm{G}}} \tilde{w} \mathrm{~d} V+\int_{V_{\mathrm{L}}}\left[\frac{1}{2} \sigma_{i j}\left(u_{i, j}+u_{j, i}\right)-w\right] \mathrm{d} V \\
& -\int_{S^{\prime}} \overline{\boldsymbol{i}}_{i} u_{i} \mathrm{~d} S
\end{aligned}
$$

where $\tilde{w}=\tilde{w}\left(u_{i}\right)$ is the strain energy density function in the global domain $V_{\mathrm{G}}, w=w\left(\sigma_{i j}\right)$ is the complementary strain energy in the local domain $V_{\mathrm{L}}$, and $\overline{\boldsymbol{t}}_{i}$ is the traction at the prescribed force boundary $S^{\prime}$.
To apply this principle to the MFEM numerical approach, identical displacement interpolation functions are used in the two domains to ensure a displacement continuity at the common boundary. The displacement field $u$ within an element (stress or displacement element) is discretized by nodal displacement parameters $q$ :

$$
u^{\mathrm{e}}=\mathbf{N} q^{\mathrm{e}}
$$

in which $\mathbf{N}$ is the displacement interpolation function matrix, and the superscripts ' $e$ ', used in the following in the same way, denote that the variables are element quantities.

A hybrid stress element is employed in the local domain to secure a good solution precision, and eqn (3) gives the stress assumption in a hybrid element:

$$
\boldsymbol{\sigma}^{\mathrm{e}}=\mathbf{P} \beta^{\mathrm{e}}
$$

where $\sigma$ and $\boldsymbol{\beta}$ are element stress and stress parameter vectors. $\mathbf{P}$ is the stress interpolation matrix which, as required by the hybrid stress element theory, ${ }^{11,12}$ guarantees the satisfaction of the homogeneous equilibrium equations by the element stress vector $\boldsymbol{\sigma}$.

Substituting eqns (2) and (3) into eqn (1) and performing variation with respect to the two sets of parameters $q$ and $\beta$ to arrive at the stationary value of the functional $F$, the following generalized system equilibrium equation is obtained:

$$
\mathbf{K} q=Q
$$

in which $\mathbf{K}$ is the overall stiffness matrix and

$$
\mathbf{K}=\mathbf{K}_{\mathrm{G}}+\mathbf{K}_{\mathrm{L}}
$$

In eqn (5) $\mathbf{K}_{\mathrm{G}}$ and $\mathrm{K}_{\mathrm{L}}$ represent the stiffness matrices of the elements in the global and local domains respectively. They are evaluated by the following expressions:

$$
\begin{aligned}
& \mathbf{K}_{\mathrm{G}}=\sum_{\mathrm{e}_{\mathrm{G}}} \mathbf{K}_{\mathrm{G}}^{\mathrm{e}}, \mathbf{K}_{\mathrm{G}}^{\mathrm{e}}=\int_{V_{\mathrm{G}}^{\mathrm{e}}} \mathbf{B}^{\mathrm{T}} \mathbf{D B} \mathrm{d} V \\
& \mathbf{K}_{\mathrm{L}}=\sum_{\mathrm{e}_{\mathrm{L}}} \mathbf{K}_{\mathrm{L}}^{\mathrm{e}}, \mathbf{K}_{\mathrm{L}}^{\mathrm{e}}=\left(\mathbf{G}^{\mathrm{T}} \mathbf{H G}\right)^{\mathrm{e}} \\
& \mathbf{G}^{\mathrm{e}}=\int_{V_{\mathrm{L}}} \mathbf{P}^{\mathrm{T}} \mathbf{B} \mathrm{d} V, \mathbf{H}^{\mathrm{e}}=\int_{V_{\mathrm{L}}} \mathbf{P}^{\mathrm{T}} \mathbf{S P} \mathrm{d} V
\end{aligned}
$$

In eqns (6) and (7), B is the strain-displacement matrix, and $\mathbf{D}, \mathbf{S}$ are the stiffness and compliance matrices of the material in the laminate coordinate system. It should be noted that the summations in eqns $(5)-(7)$ for the stiffness matrices represent 
the assembly of matrices determined by the element connectivity.

\section{$3 \quad 49 \beta-42 q$ MFEM FORMULATION}

Having arrived at the general formulae of the mixed finite element method (eqns (2)-(7)), displacement and stress interpolation functions $N$ and $P$ are still to be selected for a converged solution. In the following, a specific $49 \beta-42 q$ MFEM formulation is introduced.

\subsection{Element geometry}

The mixed finite element given here are constructed for the solution of laminate plates with planar mid-surfaces. Thus the discretization can be achieved by using prismatic elements with triangular cross-sections as shown in Fig. 1. In the figure, $a$ and $b$ are the top and bottom surfaces of the element, each having six nodes as illustrated, and $c$ is the middle surface of the element with six nodes. Three degrees of freedom $u, v$ and $w$ are assigned to each of the 12 nodes on surfaces $a$ and $b$, while only one transverse displacement degree of freedom is given to each of the six nodes on surface $c$. A total of 42 displacement degrees of freedom per element is accordingly produced.

\subsection{Stress interpolation function for the hybrid stress element}

The form of the stress interpolation matrix $\mathbf{P}$ must be chosen subject to several conditions. First, the stress field by the interpolation function needs to satisfy the equilibrium conditions. ${ }^{12}$ Apart from this, complete polynomials about $x$ and $y$ are needed to eliminate possible occurrence of spurious asymmetric displacement modes. ${ }^{13}$ Finally, a reinforcement of an interlaminar stress continuity at the interface between two adjacent layers of elements is favoured to avoid stress mismatch at laminar interfaces. ${ }^{5}$

With these restrictions taken into consideration, the stress interpolation matrix $P$ in eqn. (3) is constructed and the detailed expressions of the stresses are given in eqn (8). The formulae are obtained by expressing the stress field in terms of in-plane force resultants $N_{x}, N_{y}, N_{x y}$ (see eqn (8) in Ref. 8), and then interpolating these with polynomials of $x$ and $y$.

$$
\begin{aligned}
\sigma_{x}= & \frac{1}{2 t}\left[\beta_{10}+\left(\beta_{4}-\beta_{44}-\beta_{18}\right) x+\beta_{11} y\right. \\
& +\frac{1}{2}\left(\beta_{5}-\beta_{45}-\beta_{20}\right) x^{2}+\left(\beta_{6}-\beta_{46}-2 \beta_{21}\right) x y \\
& \left.+\beta_{12} y^{2}\right]+\frac{3}{2 t^{2}} \xi\left[\beta_{22}+\beta_{23} x+\beta_{24} y+\beta_{25} x^{2}\right. \\
& \left.+\beta_{26} x y+\beta_{27} y^{2}\right] \\
\sigma_{y}= & \frac{1}{2 t}\left[\beta_{13}+\left(\beta_{1}-\beta_{41}-\beta_{47}\right) y+\beta_{14} x\right. \\
& +\frac{1}{2}\left(\beta_{3}-\beta_{43}-\beta_{20}\right) y^{2}+\left(\beta_{2}-\beta_{42}-2 \beta_{19}\right) x y \\
& \left.+\beta_{15} x^{2}\right]+\frac{3}{2 t^{2}} \xi\left[\beta_{28}+\beta_{29} x+\beta_{30} y\right. \\
& \left.+\beta_{31} x^{2}+\beta_{32} x y+\beta_{33} y^{2}\right]
\end{aligned}
$$

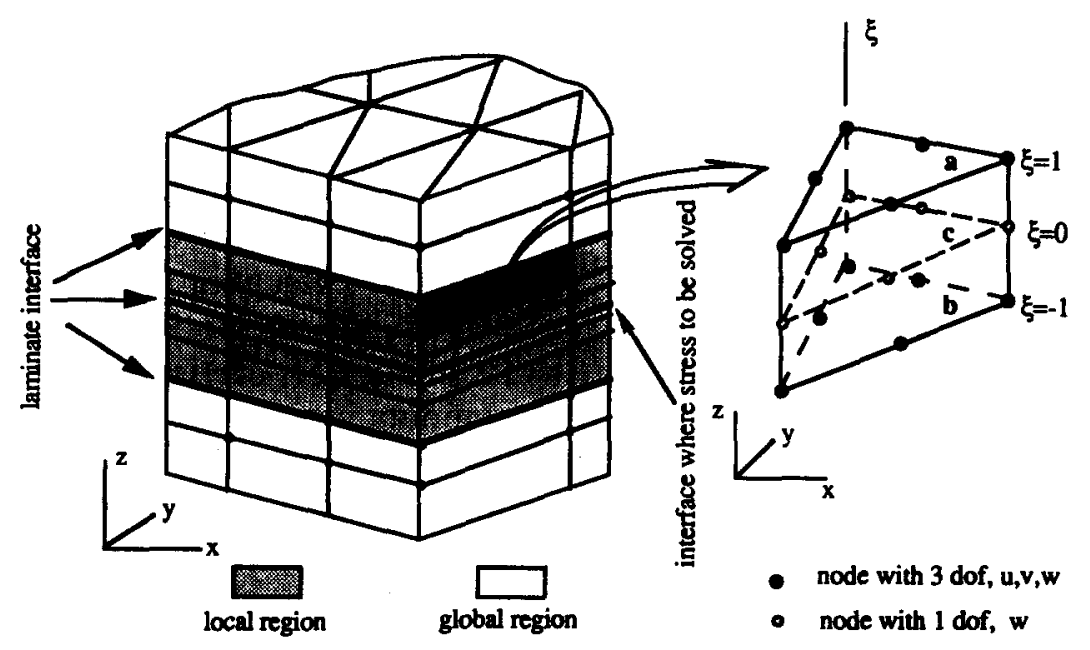

Fig. 1. Illustration of element geometry and laminate discretization. 


$$
\begin{aligned}
& \sigma_{x y}=\frac{1}{2 t}\left[\beta_{16}+\beta_{17} x+\beta_{18} y+\beta_{19} x^{2}+\beta_{20} x y\right. \\
& \left.+\beta_{21} y^{2}\right]+\frac{3}{2 t^{2}} \xi\left[\beta_{34}+\beta_{35} x+\beta_{36} y+\beta_{37} x^{2}\right. \\
& +\frac{1}{2}\left(\beta_{7}-\beta_{47}-2 \beta_{25}-2 \beta_{33}\right) x y \\
& -\frac{t}{2}\left(\beta_{3}+\beta_{43}+\beta_{5}+\beta_{45}\right) x y+\beta_{38} y^{2}+\beta_{39} x^{3} \\
& \left.+\frac{1}{4}\left(\beta_{8}-\beta_{48}\right) x^{2} y+\frac{1}{4}\left(\beta_{9}-\beta_{49}\right) x y^{2}+\beta_{40} y^{3}\right] \\
& \sigma_{y z}=\frac{3}{4 t}\left[\left(\beta_{30}+\beta_{35}+\left(\beta_{32}+2 \beta_{37}\right) x+2 \beta_{33} y\right)\right. \\
& +\frac{2 t}{3}\left(\beta_{1}+\beta_{41}\right)+\frac{2 t}{3}\left(\beta_{2}+\beta_{42}\right) x \\
& +\frac{2 t}{3}\left(\beta_{3}+\beta_{43}\right) y+\frac{1}{2}\left(\beta_{7}-\beta_{47}-2 \beta_{25}-2 \beta_{33}\right) y \\
& -\frac{t}{2}\left(\beta_{3}+\beta_{43}+\beta_{5}+\beta_{45}\right) y+3 \beta_{39} x^{2} \\
& \left.+\frac{1}{2}\left(\beta_{8}-\beta_{48}\right) x y+\frac{1}{4}\left(\beta_{9}-\beta_{49}\right) y^{2}\right] \\
& +\xi\left[\frac{1}{2}\left(\beta_{41}-\beta_{1}\right)+\frac{1}{2}\left(\beta_{42}-\beta_{2}\right) x\right. \\
& \left.+\frac{1}{2}\left(\beta_{43}-\beta_{3}\right) y\right]-\frac{3}{4 t} \xi^{2}\left[\left(\beta_{30}+\beta_{35}\right.\right. \\
& \left.+\left(\beta_{32}+2 \beta_{37}\right) x+2 \beta_{33} y\right)+\frac{1}{2}\left(\beta_{7}-\beta_{47}\right. \\
& \left.-2 \beta_{25}-2 \beta_{33}\right) y-\frac{t}{2}\left(\beta_{3}+\beta_{43}+\beta_{5}+\beta_{45}\right) y \\
& \left.+3 \beta_{39} x^{2}+\frac{1}{2}\left(\beta_{8}-\beta_{48}\right) x y+\frac{1}{4}\left(\beta_{9}-\beta_{49}\right) y^{2}\right] \\
& \sigma_{x z}=\frac{3}{4 t}\left[\left(\beta_{23}+\beta_{36}+\left(\beta_{26}+2 \beta_{38}\right) y+2 \beta_{25} x\right)\right. \\
& +\frac{2 t}{3}\left(\beta_{4}+\beta_{44}\right)+\frac{2 t}{3}\left(\beta_{5}+\beta_{45}\right) x \\
& +\frac{2 t}{3}\left(\beta_{6}+\beta_{46}\right) y+\frac{1}{2}\left(\beta_{7}-\beta_{47}-2 \beta_{25}\right. \\
& \left.-2 \beta_{33}\right) x-\frac{t}{2}\left(\beta_{3}+\beta_{43}+\beta_{5}+\beta_{45}\right) x \\
& \left.+3 \beta_{40} y^{2}+\frac{1}{2}\left(\beta_{9}-\beta_{49}\right) x y+\frac{1}{4}\left(\beta_{8}-\beta_{48}\right) x^{2}\right] \\
& +\xi\left[\frac{1}{2}\left(\beta_{44}-\beta_{4}\right)+\frac{1}{2}\left(\beta_{45}-\beta_{5}\right) x\right.
\end{aligned}
$$

$$
\begin{aligned}
& \left.+\frac{1}{2}\left(\beta_{46}-\beta_{6}\right) y\right] \\
& -\frac{3}{4} \xi^{2}\left[\left(\beta_{23}+\beta_{36}+\left(\beta_{26}+2 \beta_{38}\right) y\right.\right. \\
& \left.+2 \beta_{25} x\right)+\frac{1}{2}\left(\beta_{7}-\beta_{47}-2 \beta_{25}-2 \beta_{33}\right) x \\
& -\frac{t}{2}\left(\beta_{3}+\beta_{43}+\beta_{5}+\beta_{45}\right) x+3 \beta_{40} y^{2} \\
& \left.+\frac{1}{2}\left(\beta_{9}-\beta_{49}\right) x y+\frac{1}{4}\left(\beta_{8}-\beta_{48}\right) x^{2}\right] \\
\sigma_{z}= & {\left[\frac{1}{2}\left(\beta_{7}+\beta_{47}\right)-\frac{t}{4}\left(\beta_{5}-\beta_{45}+\beta_{3}-\beta_{43}\right)\right.} \\
& \left.+\frac{1}{2}\left(\beta_{8}+\beta_{48}\right) x+\frac{1}{2}\left(\beta_{9}+\beta_{49}\right) y\right] \\
& +\frac{3}{4} \xi\left[\left(\beta_{47}-\beta_{7}\right)+\left(\beta_{48}-\beta_{8}\right) x+\left(\beta_{49}-\beta_{9}\right) y\right. \\
& \left.+\frac{t}{4}\left(\beta_{5}+\beta_{45}+\beta_{3}+\beta_{43}\right)\right] \\
& +\frac{3}{4} \xi^{2}\left[\beta_{5}-\beta_{45}+\beta_{3}-\beta_{43}\right] \\
& -\frac{1}{4} \xi^{3}\left[\left(\beta_{47}-\beta_{7}\right)+\left(\beta_{48}-\beta_{8}\right) x+\left(\beta_{49}-\beta_{9}\right) y\right. \\
& \left.-t\left(\beta_{3}+\beta_{43}+\beta_{5}+\beta_{45}\right)\right]
\end{aligned}
$$

In the formulae, $\xi(1 \cdot 0 \geqslant \xi \geqslant-1.0)$ is an element coordinate in the thickness direction. The stresses at the top and bottom surfaces ( $a$ and $b$ in Fig. 1) of an element are obtained when $\xi$ takes the values of 1.0 and -1.0 respectively. Therefore, the stress continuity at a certain laminar interface can be prescribed by imposing the following constraints to $\sigma_{y z}, \sigma_{x z}$ and $\sigma_{z}$ :

$$
\left(\beta_{k}\right)^{\prime}=\left(\beta_{k-40}\right)^{\mathrm{u}}(k=41 \sim 49)
$$

where the superscripts 1 and $u$ represent the layer of elements below and above the element interface, which is a laminar interface when the elements lie in two adjacent laminae.

In the evaluation of the $\mathbf{H}$ and $\mathbf{G}$ matrices in eqn (6), the integration in the thickness direction, i.e. about $\xi$, is carried out explicitly to reduce the computation time. Gaussian quadrature formulae for triangles ${ }^{14}$ are used to perform the integration about $x$ and $y$. It is determined through numerical convergence experiments that a 13-point formula with an algebraic precision of seven orders is required for calculating the $\mathbf{H}$ and $\mathbf{G}$ matrices, whereas a nine-point, five-order quadra- 
ture formula is needed for the evaluation of the displacement element stiffness matrix $\left(K_{G}\right.$ in eqn $(6))$.

\subsection{Displacement interpolation function}

Six-node iso-parametric triangular interpolation functions are employed for the in-plane $(x-0-y$ plane) distribution of the displacements. In the thickness direction ( $z$ direction) a Lagrangian interpolation is applied. The resulting displacement interpolation formulae are:

$$
\begin{aligned}
{\left[\begin{array}{l}
u \\
v
\end{array}\right]=} & \frac{1-\xi}{2} \sum_{i=1}^{6} N_{i}\left[\begin{array}{c}
u_{i}^{a} \\
v_{i}^{a}
\end{array}\right\}+\frac{1+\xi}{2} \sum_{i=1}^{6} N_{i}\left\{\begin{array}{c}
u_{i}^{b} \\
v_{i}^{b}
\end{array}\right\} \\
w= & \frac{\xi(1-\xi)}{2} \sum_{i=1}^{6} N_{i} w_{i}^{a}+\frac{\xi(1+\xi)}{2} \sum_{i=1}^{6} N_{i} w_{i}^{b} \\
& +\left(1-\xi^{2}\right) \sum_{i=1}^{6} N_{i} w_{i}^{c}
\end{aligned}
$$

where $\xi$ is the normalized element transverse coordinate defined by

$$
\xi=\frac{z-z_{0}}{t}, z_{0}=\frac{1}{2}\left(z^{a}+z^{b}\right), t=\frac{1}{2}\left(z^{a}-z^{b}\right)
$$

with $z_{0}$ being the $z$ coordinate of plane $c$; and $z^{\prime \prime}$, $z^{b}$ those of planes $a$ and $b$ (see Fig. 1).

In eqn (10), the nodal displacements with superscripts stand for the displacements at the corresponding surfaces, and $N_{i}(i=1,6)$ are the in-plane displacement interpolation functions, with area coordinates $L_{1}, L_{2}$ and $L_{3}$ being a transformation of the $x-y$ coordinate system defined by:

$$
\begin{aligned}
& N_{i}=L_{i}\left(2 L_{i}-1\right) \quad i=1,2,3 \\
& L_{4}=4 L_{1} L_{2}, L_{5}=4 L_{2} L_{3}, L_{4}=4 L_{1} L_{3}
\end{aligned}
$$

\section{HYBRID STRESS ELEMENT STIFFNESS MATRIX SPECTRAL ANALYSIS}

Since a dual-variable hybrid stress element appears in the present MFEM, a careful inspection of the element quality is necessary to assess the compatibility between the stress and displacement fields assumed. An eigenvalue analysis (or spectral analysis) of the stress element stiffness matrix was conducted, and the results confirmed the matching of the parameters.
The stress and displacement parameter matching conditions can be physically interpreted as a requirement for the existence of general work between the stress and strain fields. Mathematically, they are equivalent to the possession of an appropriate eigen condition by the stiffness matrix. This eigen-condition ${ }^{15}$ requires that the number of zero eigenvalues of an element stiffness matrix be equal to that of the zero strain energy displacement modes, i.e. the number of the rigid body motion modes of the mechanics model itself; and that the corresponding eigenvectors reflect rigid body displacement modes.

The necessary condition ensuring a correct rank of the stiffness matrix is: ${ }^{15}$

$$
n_{\beta} \geqslant n_{q}-L
$$

where $L$ denotes the rigid body displacement degree of freedom, $n_{\beta}$ and $n_{q}$ are the numbers of the stress and displacement parameters, respectively. Unlike the necessary condition, the sufficient condition ${ }^{15}$ is not available in general form.

In the present work, a rank assessment after stiffness matrix derivation was used to check the rank condition of the hybrid stress element stiffness matrix. This was achieved by numerically evaluating the eigenvalues of the stiffness matrix after the derivation of element stiffness matrix, thus avoiding the difficulty in finding the sufficient condition for the correct rank.

During the course of this investigation, complying with the requirements on the $\mathbf{P}$ matrix (see Section 3.2), three stress interpolations with $44 \beta$, $49 \beta$ and $57 \beta$ were selected to couple the $42 q$ displacement field. These were all subjected to the rank assessments. In a three-dimensional elastic problem, the rigid body movement degree of freedom is 6 , therefore all the three candidates satisfy eqn (13). The $44 \beta$ formula was rejected because the element stiffness matrix possessed 11 zero eigenvalues, five more than the correct number. The $49 \beta$ and $57 \beta$ models both featured six zero eigenvalues, but the latter was also ruled out since a subsequent numerical test on a thick plate bending problem showed that it had excessive flexibility.

A unit height, equilateral triangular crosssectional (the length of all the nine sides being $1.00)$ element geometry was chosen for the stress element spectral analysis, which was carried out by using a Jacobi algorithm. To simulate isotropic material and unidirectional composite cases, two sets of nondimensionalized material properties were used. These were $E=2 \cdot 1, \nu=0.31$ for the 
3-dimensional view
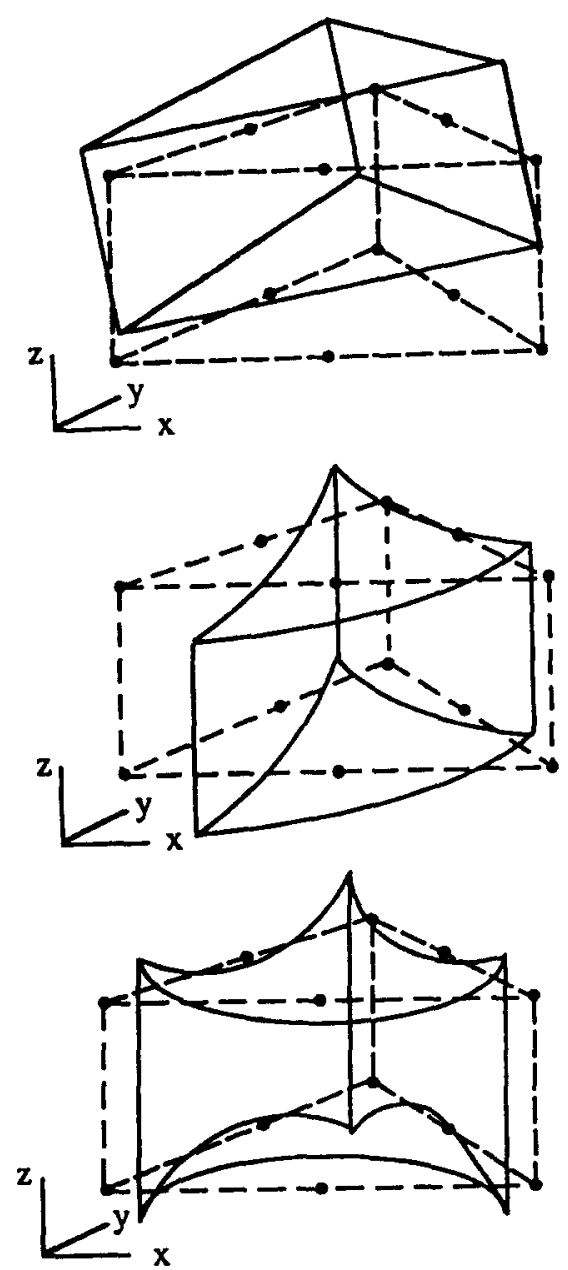

front view

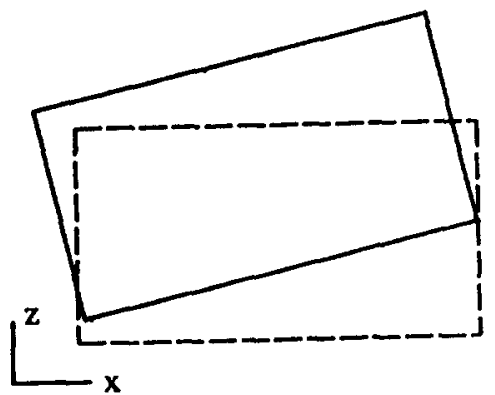

(a)

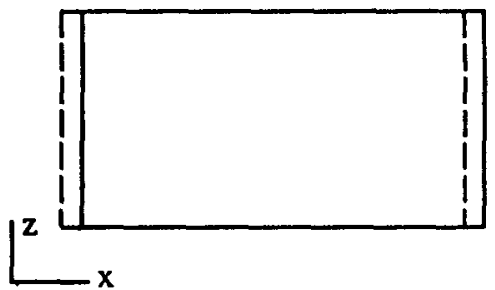

(b)

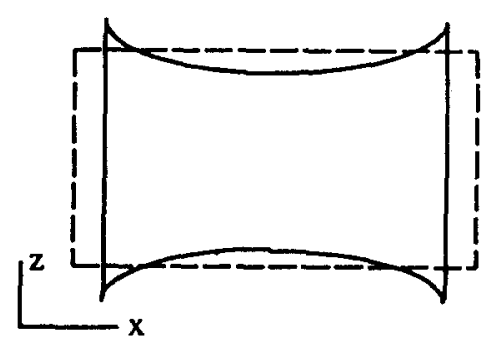

(c) top view

(figures showing displacement

in $\mathrm{z}$ direction )
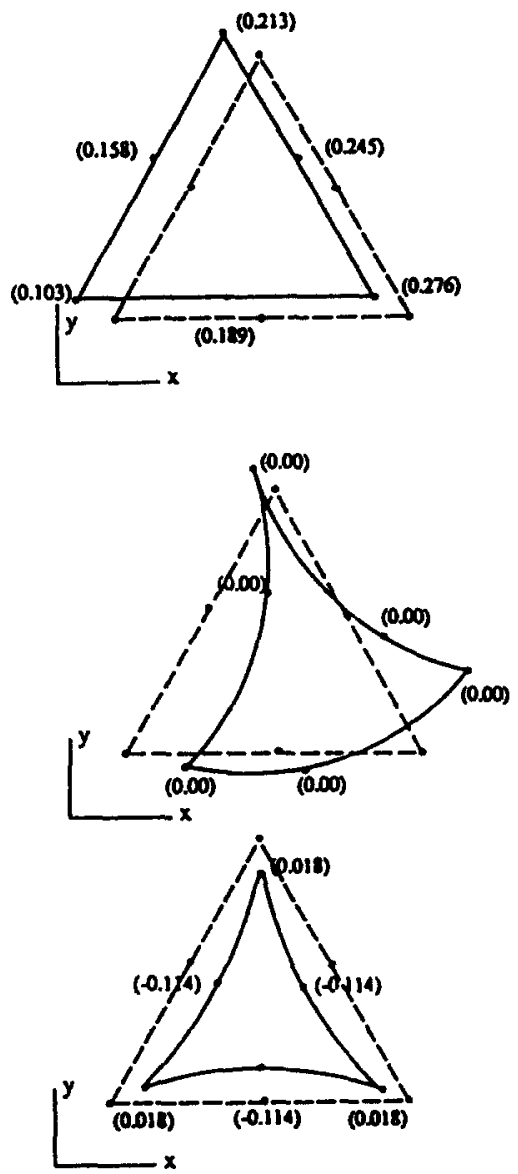

Fig. 2. Illustration of three typical eigenvalues and corresponding displacement modes, (a) $\lambda=0.00000,(b) \lambda=4 \cdot 664108$, (c) $\lambda=16.66879$.

isotropic case and $E_{1}=20, E_{2}=2 \cdot 1, G_{12}=0 \cdot 85$ and $v_{12}=v_{23}=0 \cdot 21$ for the anisotropic. The stiffness matrix of the $49 \beta$ element gave six zero eigenvalues with six associated eigenvectors reflecting rigid body motion displacement modes. The Appendix shows the eigenvalue results of the isotropic case. Figure 2 illustrates three representative pairs of eigenvalues and vectors of the isotropic case, (a) shows a rigid body mode, and (b) and (c) show two higher order deformation modes.

\section{NUMERICAL EXAMPLES AND DISCUSSION}

Two typical interlaminar stress problems were chosen to examine the applicability of the MFEM approach and the $49 \beta-42 q$ formulation proposed in this paper.

\subsection{Example 1: Cylindrical bending of thick laminated composite plate}

This problem has been solved by analytical ${ }^{16}$ and finite element methods. ${ }^{5}$ It was computed here using separately the hybrid stress and displacement elements to check the individual convergences of the elements by examining the displacement results. The $[0 / 90 / 0]$ laminate shown in Fig. 3(a) is subjected to a sinusoidal distribution load on the top surface and simply supported at the $x=0$ and $x=L$ edges. The laminar material properties used are listed in Table 1, the same as those in Ref. 5 and 16 for comparison reasons. To apply the three-dimensional MFEM 


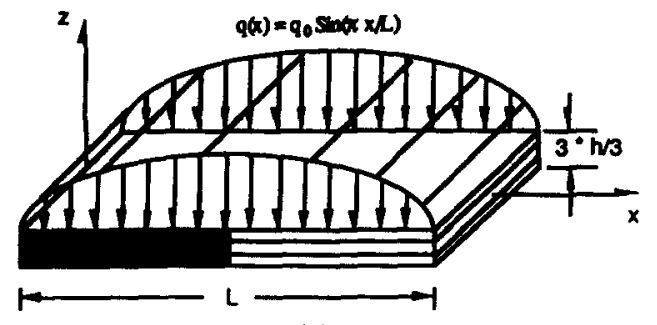

(a)

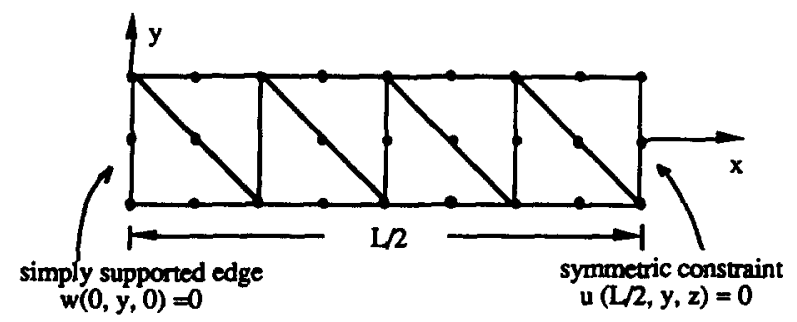

(b)

Fig. 3. Cylindrical bending problem and MFEM mesh; (a) illustration of cylindrical bending problem, (b) in-plane mesh division for the MFEM calculation.

Table 1. Material properties used in the numerical examples

\begin{tabular}{lcccccc}
\hline & $E_{11}$ & $E_{22}$ & $G_{12}$ & $G_{23}$ & $v_{23}$ & $v_{12}$ \\
\hline Example 1 & 172.37 & 6.89 & 3.44 & 1.38 & $0 \cdot 25$ & $0 \cdot 25$ \\
Example 2 & 137.89 & 14.48 & 5.86 & $5 \cdot 86$ & $0 \cdot 21$ & 0.21 \\
\hline
\end{tabular}

Unit for moduli: GPa.

to the two-dimensional problem, a strip with a width of $L / 8$ was taken out from the infinitely long laminate, and only a half of the strip was modelled as shown in Fig. 3(b) due to symmetry. At the $y= \pm L / 16$ sides, a boundary condition of $v(x, \pm L / 16, z)=0$ was assumed to stimulate the constraint.

The in-plane mesh division is shown in Fig. 3 (b). In both the stress and displacement element calculations, each lamina was modelled by a layer of elements with the whole plate represented by three layers of elements. The convergences of the mid-plane maximum deflection results by the two types of elements are shown in Fig. 4. The ordinate in the figure is a relative error defined by:

$$
E=\left(\bar{W}_{\text {MFEM }}-\bar{W}_{\text {exact }}\right) / \bar{W}_{\text {exact }}
$$

where $\bar{w}_{\text {MFEM }}$ and $\bar{w}_{\text {exact }}$ are nondimensionalized maximum mid-plane deflections by MFEM and elasticity, ${ }^{16}$ respectively.

It is shown that the solution from both kinds of elements converged to the exact solution with the mesh refinement, but from different directions.

To check the ability of the elements to reflect transverse shear deformation, the maximum mid-

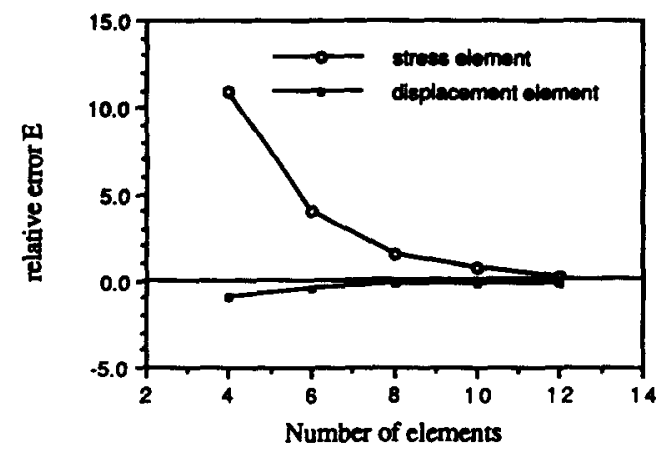

Fig. 4. Convergence of mid-plane maximum deflection by stress and displacement elements, respectively.

Table 2. Mid-plane maximum defiection $W$ at different relative span $h \boldsymbol{L}$

\begin{tabular}{ccccc}
\hline$h / L$ & $W_{\text {exact }}{ }^{a}$ & $\begin{array}{c}\text { CLT } \\
\text { prediction }\end{array}$ & $W_{\mathrm{G}{ }^{a}}$ & $W_{\mathrm{L}}{ }^{a}$ \\
\hline 0.01 & 0.513 & $(0.993)^{b}$ & $0.512(0.998)$ & - \\
0.05 & 0.617 & $(0.826)$ & $0.612(0.992)$ & - \\
0.10 & 0.933 & $(0.546)$ & $0.924(0.990)$ & $0.947(1.015)$ \\
0.25 & 2.887 & $(0.177)$ & $3.000(1.040)$ & $3.200(1.108)$ \\
\hline
\end{tabular}

" $W_{\text {exact }}$ represents the exact elasticity solution; $W_{\mathrm{G}}$ and $W_{\mathrm{L}}$ from stress and displacement, respectively.

${ }^{b}$ Figures in brackets represent the ratio of the item to $W_{\text {exacl. }}$.

plane deflections of laminates with relative spans $(h / L)$ from 0.01 to 0.25 were computed using the two kinds of elements separately. Table 2 gives a comparison between the computed values, classical lamination theory prediction and the exact elasticity solution. Eight elements were used to model the plate in the $x-y$ plane. Both the local region based stress and the global region based displacement elements are seen to be capable of modelling thick laminates with transverse shear deformation, which is expected from the high order interpolation functions.

\subsection{Example 2: Free-edge interlaminar stress problem}

The Pagano free-edge interlaminar stress problem ${ }^{1,17}$ with $[0 / 90 / 90 / 0]$ symmetric laminate with a half-width to thickness ratio of 8 was also solved by the MFEM of this paper. The material constants $^{1,17}$ adopted are listed in Table 1.

In the calculation, only a quarter of the plate shown in Fig. 5(a) was modelled by the MFEM due to symmetry, and an in-plane mesh arrangement similar to that for Example (1) was adopted. Two through-thickness mesh division schemes, as shown in Fig. 5(b), were employed in the thick- 


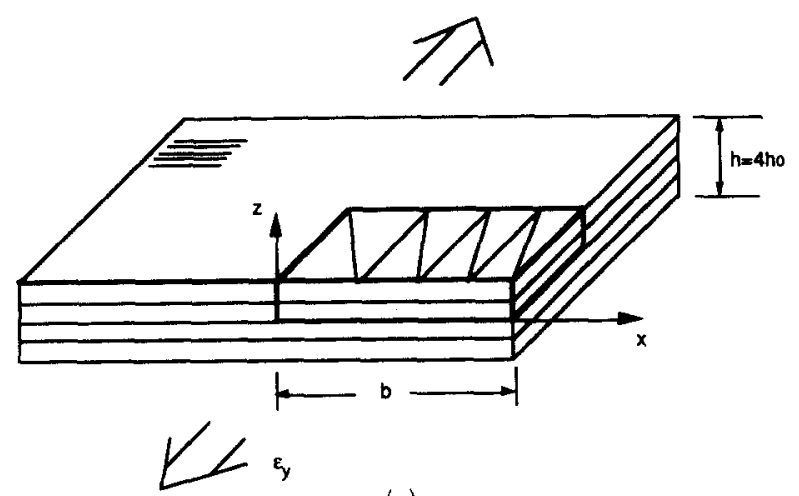

(a)

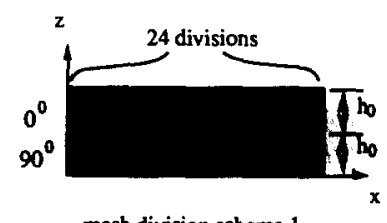

mesh division scheme 1

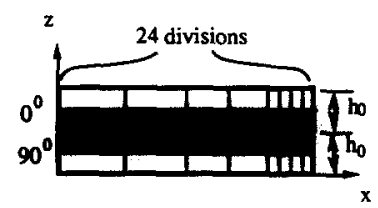

mesh division scheme 2 local region, stress element
global region, disp. element

(b)

Fig. 5. Free-edge stress problem and mesh division schemes; (a) geometry of the free-edge problem, (b) two mesh divisions for the free edge problem modelling.

ness of the laminate. The first was a pure stress element division, with four layers of elements included to model the $0^{\circ}$ and $90^{\circ}$ layer. In the second, a global-local-global MFEM division was used with two layers of stress elements at different sides of the $0 / 90$ interface. A total of 2850 degrees of freedom was involved in the modelling. This was a result of a 48 element discretization of the region, with the elements near the free-edge $x=b$ having a width to height ratio of $0 \cdot 5$.

An examination of the stress solution under the first mesh division scheme, which was expected to produce a rather good prediction as a result of the use of stress element in the entire region, was carried out. Figure 6 shows the comparison of the results of the interlaminar normal stress $\sigma_{z}$ at the interface $z=0$ with a conventional finite element analysis ${ }^{17}$ in the literature. It is seen that even with this four-layer stress element modelling, the interlaminar stress prediction gave an acceptable precision.

The second mesh division scheme was employed to investigate the interlaminar stress prediction by the MFEM approach. It was found through the stress results that a good interlaminar stress prediction was produced in the stress element modelled local region, while a less precise result was yielded in the displacement modelled global region. The distribution of the interlaminar normal stress $\sigma_{z}$ at $z=0$ and $z=h$ by the all-stress

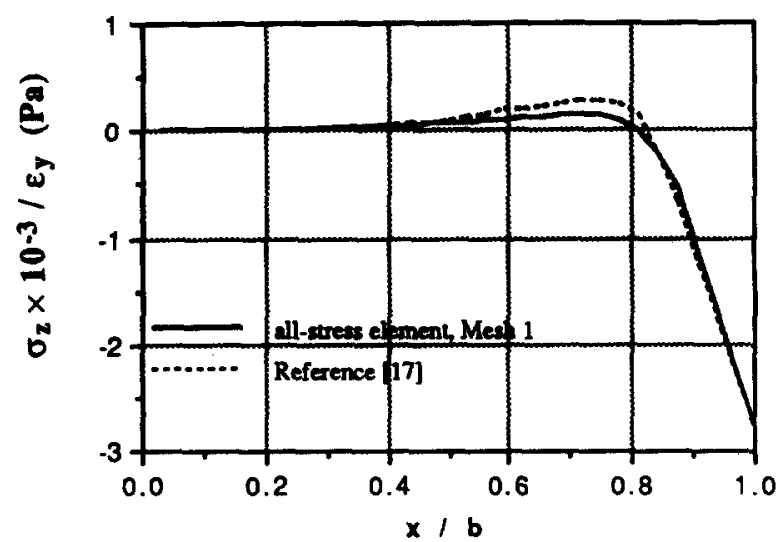

Fig. 6. Comparison of interlaminar normal stress $\sigma_{z}$ predictions at $z=0$ by MFEM (all-stress element) and ordinary FEM. $^{17}$

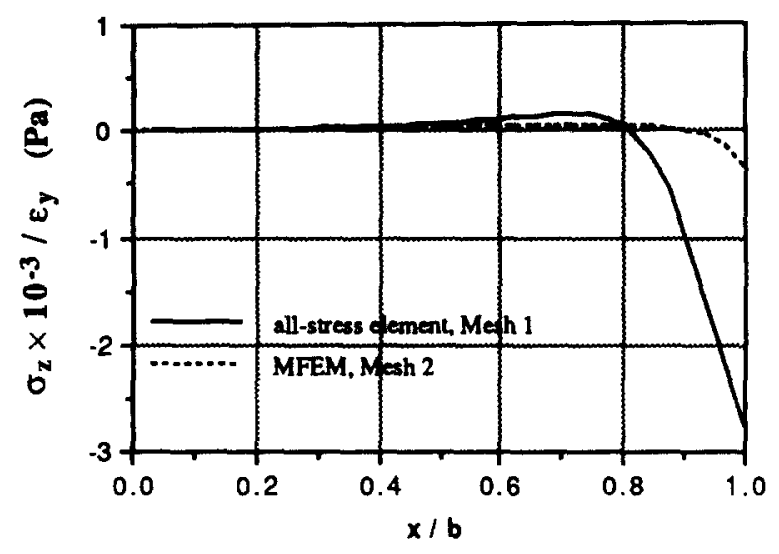

Fig. 7. Comparison of $\alpha_{z}(z=0)$ by all-stress element and MFEM mesh divisions.

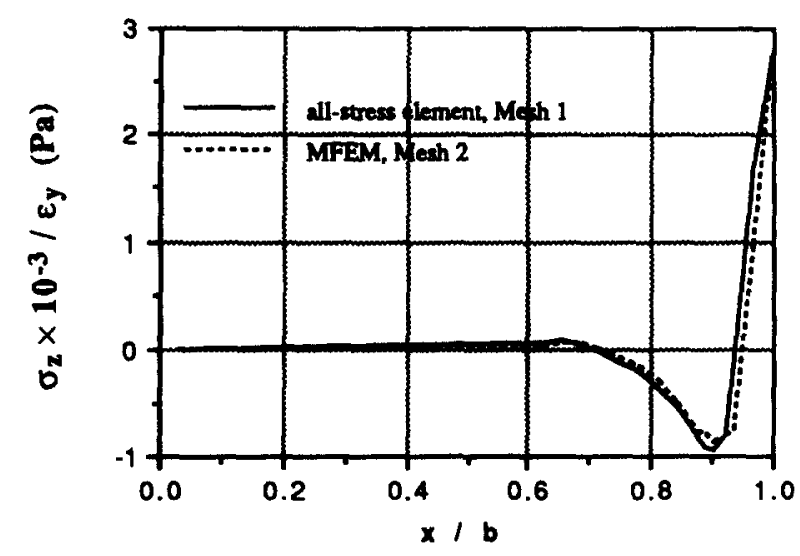

Fig. 8. Comparison of $\sigma_{z}\left(z=h_{0}, 0 / 90\right.$ interface $)$ by allstress element and MFEM mesh divisions.

element modelling (the first mesh division) are represented by the solid lines in Figs. 7 and 8 . They are used as a baseline to assess the same stress by the MFEM (the second mesh division) scheme, shown by the dotted lines in the figures. In Fig. 7, discrepancy exists between the two curves; however, a good agreement is seen between those in Fig. 8. This fact suggests that the 
interlaminar stress was better modelled in the stress element represented local region.

\subsection{Discussion}

Numerical example 2 shows that the satisfactory stress results in the local domain can be acquired by using the MFEM, although the stress in the remaining part is less accurate. As a preliminary investigation, the examples have shown the applicability of the MFEM approach and the quality of the current formulation.

The objective of the present work is to establish a finite element approach that offers a balance between efficiency and accuracy. This has been partially achieved by reducing the degrees of freedom needed to model a laminate by incorporating displacement elements that can span several laminae. But considerable computation time is incurred by the adoption of the stress element in the current formulation. This needs to be resolved by judicious through-thickness global-local domain arrangement to minimize the use of the stress element.

\section{CONCLUSIONS}

(1) The proposed MFEM approach is feasible for three dimensional interlaminar stress analysis of composite laminates.

(2) The $49 \beta$ hybrid stress element in this MFEM has co-ordinate invariance and a correct rank, and is accurate for analysis of interlaminar stresses in composite laminate problems considered herein.

(3) The $49 \beta-42 q$ MFEM formulation is capable of producing converged solution in the three-dimensional laminate analysis, and a good stress prediction is obtainable in the stress element discretized local region. The feasibility of the MFEM approach is also proved by the examples.

\section{ACKNOWLEDGEMENT}

Support of the Chinese National Natural Science Foundation is acknowledged by the authors.

\section{REFERENCES}

1. Pipes, R. B. \& Pagano, N. J., Interlaminar stress in composite laminates under uniform axial extension, $J$. Composite Materials, 4(1970) 538-48.

2. Whitcomb, J. D. \& Raju, I. S., Analysis of interlaminar stress in thick composite laminates with and without edge delamination. In Delamination and Debonding of Materials, ed. W. S. Johnson. ASTM STP 876, American Society for Testing and Materials, Philadelphia, PA, 1985, pp. 69-94

3. Nishioka, T. \& Alturi, S. N., Stress analysis of holes in angle-ply laminates: An efficient assumed stress special hole element approach and a simple estimation method. Computers and Structures, 15 (1982) 135-47.

4. Spilker, R. L., A hybrid stress finite element formation for thick multi-layer laminates. Computers and Structures, 11 (1980) 507-14.

5. Spilker, R. L., Hybrid stress eight node elements for thin and thick multi-layer laminated plates. Int. J. Numerical Methods in Engineering, 18 (1982) 801-28.

6. Wang, A. S. D. \& Crossman, F. W., Calculation of edge stress in multilayer laminates by sub-structuring. $J$. Composite Materials, 12 (1979) 76-83.

7. Jones, R., Callinan, R., Teh, K. K. \& Brown, K. C., Analysis of multi-layer laminates using three-dimensional super-elements. Int. J. Numerical Methods in Engineering, 20 (1984) 583-7.

8. Pagano, N. J., Stress fields in composite materials. Int. J. Solids and Structures, 14 (1978) 385-400.

9. Pagano, N. J. \& Soni, S. R., Global-local laminate variational model. Int. J. Solids and Structures, 19 (1983) 207-28.

10. Soni, S. R. \& Kim, R. Y., Analysis of suppression of the free-edge delamination by introducing adhesive layer. In Proc. ICCM VI \& ECCM II, London, 20-24 July, 1987, ed. F. L. Matthews et al., Elsevier Applied Science, London, pp. 5.219-29.

11. Pian, T. H. H., Derivation of element stiffness matrices by assumed stress distributions. AIAA Journal, 2 (1964) 1333-6.

12. Tong, P. \& Pian, T. H. H., A variational principle and convergence of a finite method based on assumed stress distribution. Int. J. Solids and Structures, 5 (1969) 463-72.

13. Cook, R. D., Two hybrid elements for analysis of thick, thin and sandwich plates, Int. J. Numerical Methods in Engineering, 5 (1972) 277-88.

14. Cowper, G. R., Gaussian quadrature formulas for triangles. Int. J. Numerical Methods in Engineering, 7 (1973) 405-8.

15. Pian, T. H. H. \& Chen, D. P., Zero energy deformation modes in assumed stress finite elements. Int. J. Numerical Methods in Engineering, 9 (1983) 1741-52.

16. Pagano, N. J., Exact solutions for composite laminates in cylindrical bending, J. Composite Materials, 3 (1964) 398-411.

17. Wang, A. S. D. \& Crossman, F. W., Some new results on edge effect in symmetric composite laminates. J. Composite Materials, 11 (1977) 92-106. 


\section{APPENDIX}

Table A1. Results of Eigenvalue Examination of the Hybrid Stress Element

\begin{tabular}{llllll}
\hline 0.000000 & 0.000000 & 0.000000 & 0.000000 & $0 \cdot 000000$ & $0 \cdot 000000$ \\
0.048218 & 0.051270 & 0.108551 & $0 \cdot 115861$ & $0 \cdot 130069$ & $0 \cdot 201689$ \\
0.235727 & 0.240453 & 0.240469 & 0.312823 & 0.445939 & 0.463181 \\
0.485285 & 0.580939 & 0.581011 & 0.785120 & 0.792992 & 0.924008 \\
0.991785 & $1 \cdot 145845$ & 1.329062 & $1 \cdot 329216$ & 1.434815 & 1.435088 \\
1.577305 & $2 \cdot 131319$ & $2 \cdot 433420$ & $2 \cdot 469790$ & 2.528787 & 4.508479 \\
4.509558 & 4.651850 & 4.667411 & 6.827389 & 6.828921 & 16.66879 \\
\hline
\end{tabular}

Note: (a) data in the table were from a unit equilateral triangular prismatic element; (b) nondimensional isotropic material property used: $E=2 \cdot 1, v=0 \cdot 31$. 